\title{
A PRIMAL-DUAL INTERIOR-POINT ALGORITHM BASED ON A NEW KERNEL FUNCTION
}

\author{
G. M. CHO ${ }^{凶 1}$, Y. Y. $\mathrm{CHO}^{2}$ and Y. H. $\mathrm{LEE}^{3}$
}

(Received 29 January, 2009; revised 5 October, 2010)

\begin{abstract}
We propose a new primal-dual interior-point algorithm based on a new kernel function for linear optimization problems. New search directions and proximity functions are proposed based on the kernel function. We show that the new algorithm has $\mathcal{O}(\sqrt{n} \log n \log (n / \epsilon))$ and $\mathcal{O}(\sqrt{n} \log (n / \epsilon))$ iteration bounds for large-update and small-update methods, respectively, which are currently the best known bounds for such methods.
\end{abstract}

2000 Mathematics subject classification: primary 90C05; secondary 90C51.

Keywords and phrases: primal-dual interior-point method, kernel function, complexity, polynomial algorithm, linear optimization problem.

\section{Introduction}

In this paper we propose a new primal-dual interior-point method (IPM) for the following standard linear optimization (LO) problem:

$$
\min \left\{c^{T} x: A x=b, x \geq 0\right\},
$$

where $A \in \mathbf{R}^{m \times n}, c, x \in \mathbf{R}^{n}$, and $b \in \mathbf{R}^{m}$. Without loss of generality, we assume that $m \leq n$ and $\operatorname{rank}(A)=m$. The dual problem to $(1.1)$ is

$$
\max \left\{b^{T} y: A^{T} y+s=c, s \geq 0\right\},
$$

where $y \in \mathbf{R}^{m}$ and $s \in \mathbf{R}^{n}$.

Since Karmarkar's paper [13] in 1984, IPMs have shown their efficiency in solving large-scale linear programming problems with a wide variety of successful applications. In this paper we propose a new primal-dual interior-point algorithm

\footnotetext{
${ }^{1}$ Department of Multimedia Engineering, Dongseo University, Busan 617-716, South Korea; e-mail: gcho@dongseo.ac.kr.

${ }^{2}$ Department of Mathematics, Pusan National University, Busan 609-735, South Korea; e-mail: youyoung@pusan.ac.kr.

${ }^{3}$ Department of Mathematics, Pusan National University, Busan 609-735, South Korea; e-mail: yhlee@pusan.ac.kr.

(C) Australian Mathematical Society 2011, Serial-fee code 1446-1811/2011 \$16.00
} 
which is the most efficient from a computational point of view [3]. It is generally agreed that the iteration complexity of the algorithm is an appropriate measure for its efficiency [10].

Most polynomial-time interior-point algorithms for $\mathrm{LO}$ are based on the logarithmic kernel function [3, 9, 12, 19]. Peng et al. [15, 16] proposed a new variant of IPMs based on self-regular kernel functions and achieved so far the best known complexity, $\mathcal{O}(\sqrt{n} \log n \log (n / \epsilon))$ for large-update methods with a specific self-regular function. Roos et al. [4-8, 10, 11] proposed new primal-dual IPMs for LO problems based on eligible kernel functions. They also proposed a scheme for analysing the algorithms based on four conditions on the kernel function [5] and obtained an $\mathcal{O}(\sqrt{n} \log (n / \epsilon))$ iteration bound for small-update IPMs in all cases and an $\mathcal{O}(\sqrt{n} \log n \log (n / \epsilon))$ iteration bound for large-update methods with a specific kernel function [4]. At present, the open question in this area is the existence of a kernel function whose complexity bound for large-update methods is the same as or better than the best known complexity result. Recently, Amini and Haseli [1] proposed a generalized version of the kernel function in [5], achieved the best known iteration bound for large-update methods for LO and extended to linear complementarity problems [2]. Motivated by their work, we define a new kernel function and propose a new primaldual interior-point algorithm for LO based on this kernel function. We analyse the complexity for large-update and small-update methods based on the kernel function and obtain $\mathcal{O}(\sqrt{n} \log n \log (n / \epsilon))$ and $\mathcal{O}(\sqrt{n} \log (n / \epsilon))$ iteration bounds, respectively, which are the best known results so far.

The paper is organized as follows. In Section 2 we recall the generic IPM and the motivation for the new algorithm. In Section 3 we define a new kernel function and give its properties which are essential for complexity analysis. In Section 4 we derive the complexity result for the new algorithm.

We use the following notation throughout the paper. We let $\mathbf{R}_{+}^{n}$ and $\mathbf{R}_{++}^{n}$ denote the set of $n$-dimensional nonnegative vectors and positive vectors, respectively. For $x, s \in \mathbf{R}^{n}, x_{\min }$ and $x s$ denote the smallest component of the vector $x$ and the componentwise product of the vectors $x$ and $s$, respectively. We let $e$ denote the $n$-dimensional vector $(1,1, \ldots, 1)$. For $x, s \in \mathbf{R}_{++}^{n}$ and $\mu>0$, we define $v:=$ $\sqrt{x s / \mu}, v^{-1}:=\sqrt{\mu e /(x s)}$ whose $i$ th components are $\sqrt{x_{i} s_{i} / \mu}$ and $\sqrt{\mu /\left(x_{i} s_{i}\right)}$, respectively. We denote by $X$ the diagonal matrix obtained from a vector $x$, that is, $X=\operatorname{diag}(x)$. For $f(x), g(x): \mathbf{R}_{++} \rightarrow \mathbf{R}_{++}$, we write $f(x)=\mathcal{O}(g(x))$ if there exists a positive constant $c_{1}$ such that $f(x) \leq c_{1} g(x)$ and $f(x)=\Theta(g(x))$ if there exist positive constants $c_{2}$ and $c_{3}$ such that $c_{2} g(x) \leq f(x) \leq c_{3} g(x)$, for all $x>0$.

\section{Preliminaries}

In this section we recall some basic concepts and the generic IPM. Without loss of generality, we assume that both (1.1) and (1.2) satisfy the interior-point condition (IPC) [17], that is, there exists $\left(x^{0}, y^{0}, s^{0}\right)$ such that

$$
A x^{0}=b, x^{0}>0, \quad A^{T} y^{0}+s^{0}=c, s^{0}>0 .
$$


By the duality theorem [17, Theorem II.2], finding an optimal solution of (1.1) and (1.2) is equivalent to solving the following system:

$$
A x=b, x \geq 0, \quad A^{T} y+s=c, s \geq 0, \quad x s=0 .
$$

The basic idea of primal-dual IPMs is to replace the third equation in (2.1) by the parameterized equation $x s=\mu e$ with $\mu>0$. We now consider the following system:

$$
A x=b, x>0, \quad A^{T} y+s=c, s>0, \quad x s=\mu e .
$$

If the IPC holds, then (2.2) has a unique solution for each $\mu>0$ [14]. We denote this solution by $(x(\mu), y(\mu), s(\mu))$ and call $x(\mu)$ the $\mu$-centre of $(1.1)$ and $(y(\mu), s(\mu))$ the $\mu$-centre of (1.2). The set of $\mu$-centres, $\mu>0$, is called the central path of (1.1) and (1.2) [18]. The limit of the central path as $\mu$ goes to zero exists and since the limit point satisfies (2.1), it naturally yields the optimal solution for (1.1) and (1.2) [17]. Primal-dual IPMs follow the central path approximately and approach the solution of (1.1) and (1.2) as $\mu$ goes to zero.

For given $(x, y, s):=\left(x^{0}, y^{0}, s^{0}\right)$, by applying Newton's method to system (2.2) we have the following Newton system:

$$
A \Delta x=0, \quad A^{T} \Delta y+\Delta s=0, \quad s \Delta x+x \Delta s=\mu e-x s .
$$

Since $A$ has full row rank, (2.3) has a unique solution $(\Delta x, \Delta y, \Delta s)$ which is called the search direction [17]. By taking a step along the search direction $(\Delta x, \Delta y, \Delta s)$, one constructs a new iterate $\left(x_{+}, y_{+}, s_{+}\right)$,

$$
x_{+}=x+\alpha \Delta x, \quad y_{+}=y+\alpha \Delta y, \quad s_{+}=s+\alpha \Delta s,
$$

for some $\alpha>0$.

To motivate our new algorithm we define the following scaled vectors: for $(x, s)>0$ and $\mu>0$,

$$
d_{x}:=v \Delta x / x, \quad d_{s}:=v \Delta s / s .
$$

Using (2.5), we can rewrite system (2.3) as follows:

$$
\bar{A} d_{x}=0, \quad \bar{A}^{T} \Delta y+d_{s}=0, \quad d_{x}+d_{s}=v^{-1}-v,
$$

where $\bar{A}:=(1 / \mu) A V^{-1} X, V:=\operatorname{diag}(v)$, and $X:=\operatorname{diag}(x)$. Note that the right-hand side of the third equation in (2.6) equals the negative gradient of the logarithmic barrier function $\Psi_{l}(v)$, that is,

$$
d_{x}+d_{s}=-\nabla \Psi_{l}(v)
$$

where

$$
\Psi_{l}(v):=\sum_{i=1}^{n} \psi_{l}\left(v_{i}\right), \quad \psi_{l}(t)=\sum_{i=1}^{n}\left(\left(t^{2}-1\right) / 2-\log t\right) .
$$

We call $\psi_{l}$ the classical logarithmic kernel function of $\Psi_{l}(v)$. In this paper we replace $\psi_{l}(t)$ with a new kernel function $\psi(t)$ which will be defined in (3.2). In what follows, and throughout the paper we assume that $\tau \geq 1$. 
The generic interior-point algorithm works as follows. Assume that we are given a strictly feasible point $(x, y, s)$ which is in a $\tau$-neighbourhood of the given $\mu$-centre. Then we decrease $\mu$ to $\mu_{+}=(1-\theta) \mu$, for some fixed $\theta \in(0,1)$, and then solve the Newton system (2.3) to obtain the unique search direction. The positivity of a new iterate is ensured by an appropriate choice of the step size $\alpha$ which is defined by some line search rule. This procedure is repeated until we find a new iterate $\left(x_{+}, y_{+}, s_{+}\right)$that is in a $\tau$-neighbourhood of the $\mu_{+}$-centre, and then we let $\mu:=\mu_{+}$ and $(x, y, s):=\left(x_{+}, y_{+}, s_{+}\right)$. Then $\mu$ is again reduced by the factor $1-\theta$ and we solve the Newton system targeting at the new $\mu_{+}$-centre, and so on. This process is repeated until $\mu$ is small enough, say until $n \mu<\varepsilon$.

\section{Generic primal-dual algorithm for LO}

Input:

a threshold parameter $\tau>0$;

an accuracy parameter $\varepsilon>0$;

a fixed barrier-update parameter $\theta, \quad 0<\theta<1$;

$\left(x^{0}, s^{0}\right)$ and $\mu^{0}:=1$ such that $\Psi_{l}\left(\sqrt{x^{0} s^{0} / \mu^{0}}\right) \leq \tau$; begin

$x:=x^{0} ; \quad s:=s^{0} ; \quad \mu:=\mu^{0} ;$

while $n \mu \geq \varepsilon$ do

begin

$\mu:=(1-\theta) \mu$;

while $\Psi_{l}(v)>\tau$ do

begin

solve system (2.3) for $\Delta x, \Delta y, \Delta s$;

determine a step size $\alpha$;

$x:=x+\alpha \Delta x$;

$s:=s+\alpha \Delta s$

$y:=y+\alpha \Delta y$;

$v:=\sqrt{x s / \mu}$

end

end

end

REMARK 2.1. If $\theta$ is a constant independent of the dimension of the problem, $n$, for example $\theta=1 / 2$, then we call the algorithm a large-update method. If $\theta$ depends on $n$, for example $\theta=1 / \sqrt{n}$, then the algorithm is called a small-update method.

\section{The new kernel function}

In this section we define a new kernel function which is neither self-regular nor logarithmic and gives the best known complexity result. We call $\psi: \mathbf{R}_{++} \rightarrow \mathbf{R}_{+}$a 
kernel function if $\psi$ is twice differentiable and satisfies the following conditions:

$$
\psi^{\prime}(1)=\psi(1)=0, \quad \psi^{\prime \prime}(t)>0, t>0, \quad \lim _{t \rightarrow 0^{+}} \psi(t)=\lim _{t \rightarrow \infty} \psi(t)=\infty .
$$

Now we define a new function $\psi(t)$ as follows:

$$
\psi(t):=\frac{t^{2}-1}{2}+\frac{e^{p(g(t)-1)}-1}{p q}, \quad p \geq 1, q \geq 1, t>0,
$$

where $g(t):=e^{q\left(t^{-1}-1\right)}$. Then we have the following:

$$
\begin{aligned}
\psi^{\prime}(t)= & t-e^{p(g(t)-1)} g(t) t^{-2}, \\
\psi^{\prime \prime}(t)=1 & +e^{p(g(t)-1)} g(t) t^{-4}(p q g(t)+q+2 t), \\
\psi^{\prime \prime \prime}(t)=- & e^{p(g(t)-1)} g(t) t^{-6}\left(p^{2} q^{2} g(t)^{2}+3 p q^{2} g(t)\right. \\
& \left.+6 p q g(t) t+q^{2}+6 q t+6 t^{2}\right) .
\end{aligned}
$$

From (3.3), $\psi(t)$ is clearly a kernel function and

$$
\psi^{\prime \prime}(t)>1, \quad t>0 .
$$

In this paper, we replace the function $\Psi_{l}(v)$ in (2.7) with the function $\Psi(v)$ defined as follows:

$$
d_{x}+d_{s}=-\nabla \Psi(v),
$$

where $\Psi(v)=\sum_{i=1}^{n} \psi\left(v_{i}\right)$ with $\psi(t)$ defined by (3.2). Hence the new search direction ( $\Delta x, \Delta y, \Delta s$ ) is obtained by solving the following modified Newton system:

$$
A \Delta x=0, \quad A^{T} \Delta y+\Delta s=0, \quad s \Delta x+x \Delta s=-\mu v \nabla \Psi(v) .
$$

Note that $d_{x}$ and $d_{s}$ are orthogonal because the vector $d_{x}$ belongs to the null space and $d_{s}$ to the row space of the matrix $\bar{A}$. Since $d_{x}$ and $d_{s}$ are orthogonal, we have

$$
\Psi(v)=0 \Leftrightarrow v=\mathbf{e} \Leftrightarrow \nabla \Psi(v)=0 \Leftrightarrow d_{x}=d_{s}=0 \Leftrightarrow x=x(\mu) \text { and } s=s(\mu) .
$$

We use $\Psi(v)$ as the proximity function to measure the distance between the current iterate and the $\mu$-centre for given $\mu>0$. We also define the norm-based proximity measure $\delta(v)$ as follows:

$$
\delta(v):=\frac{1}{2}\|\nabla \Psi(v)\|=\frac{1}{2}\left\|d_{x}+d_{s}\right\| .
$$

LEMMA 3.1. For $\psi(t)$, the following results hold:

(i) $\psi(t)$ is exponentially convex, that is,

$$
\psi\left(\sqrt{t_{1} t_{2}}\right) \leq \frac{1}{2}\left(\psi\left(t_{1}\right)+\psi\left(t_{2}\right)\right), \quad t_{1}, t_{2}>0 ;
$$

(ii) $\psi^{\prime \prime}(t)$ is monotonically decreasing, $t>0$;

(iii) $t \psi^{\prime \prime}(t)-\psi^{\prime}(t)>0, t>0$;

(iv) $2\left(\psi^{\prime \prime}(t)\right)^{2}-\psi^{\prime}(t) \psi^{\prime \prime \prime}(t)>0, t>0$. 
Proof. For (i), by [16, Lemma 2.1.2], it suffices to show that $\psi(t)$ satisfies $t \psi^{\prime \prime}(t)+\psi^{\prime}(t)>0, t>0$. For $t>0$, using (3.3),

$$
t \psi^{\prime \prime}(t)+\psi^{\prime}(t)=2 t+e^{p(g(t)-1)} g(t) t^{-3}(p q g(t)+q+t)>0,
$$

with $g(t)$ defined as before. For (ii), from (3.3), $\psi^{\prime \prime \prime}(t)<0, t>0$. For (iii), using (3.3), we have for $t>0$,

$$
t \psi^{\prime \prime}(t)-\psi^{\prime}(t)=e^{p(g(t)-1)} g(t) t^{-3}(p q g(t)+q+3 t)>0 .
$$

For (iv), using (3.3), we have for $t>0$,

$$
\begin{aligned}
2\left(\psi^{\prime \prime}(t)\right)^{2}-\psi^{\prime}(t) \psi^{\prime \prime \prime}(t)=2 & +e^{p(g(t)-1)} g(t) t^{-8}\left(10 p q g(t) t^{4}+10 q t^{4}\right. \\
& +14 t^{5}+p^{2} q^{2} e^{p(g(t)-1)} g(t)^{3}+q^{2} e^{p(g(t)-1)} g(t) \\
& +2 e^{p(g(t)-1)} g(t) t^{2}+p q^{2} e^{p(g(t)-1)} g(t)^{2} \\
& +2 p q e^{p(g(t)-1)} g(t)^{2} t+2 q e^{p(g(t)-1)} g(t) t \\
& \left.+p^{2} q^{2} g(t)^{2} t^{3}+3 p q^{2} e^{p(g(t)-1)} g(t) t^{3}\right)>0 .
\end{aligned}
$$

This completes the proof.

LEMMA 3.2. For $\psi(t)$, the following results hold:

(i) $(t-1)^{2} / 2 \leq \psi(t) \leq\left(\psi^{\prime}(t)\right)^{2} / 2, t>0$;

(ii) $\psi(t) \leq(p q+q+3)(t-1)^{2} / 2, p \geq 1, q \geq 1, t \geq 1$.

PROOF. For (i), using the first condition of (3.1) and (3.4),

$$
\psi(t)=\int_{1}^{t} \int_{1}^{\xi} \psi^{\prime \prime}(\zeta) d \zeta d \xi \geq \int_{1}^{t} \int_{1}^{\xi} d \zeta d \xi=\frac{1}{2}(t-1)^{2}
$$

which proves the first inequality. The second inequality is obtained as follows:

$$
\begin{aligned}
\psi(t) & =\int_{1}^{t} \int_{1}^{\xi} \psi^{\prime \prime}(\zeta) d \zeta d \xi \leq \int_{1}^{t} \int_{1}^{\xi} \psi^{\prime \prime}(\xi) \psi^{\prime \prime}(\zeta) d \zeta d \xi \\
& =\int_{1}^{t} \psi^{\prime \prime}(\xi) \psi^{\prime}(\xi) d \xi=\int_{1}^{t} \psi^{\prime}(\xi) d \psi^{\prime}(\xi)=\frac{1}{2}\left(\psi^{\prime}(t)\right)^{2}
\end{aligned}
$$

For (ii), using Taylor's theorem and noting that $\psi(1)=\psi^{\prime}(1)=0, \psi^{\prime \prime \prime}(t)<0$, for all $t>0$, and $\psi^{\prime \prime}(1)=p q+q+3$,

$$
\begin{aligned}
\psi(t) & =\psi(1)+\psi^{\prime}(1)(t-1)+\frac{1}{2} \psi^{\prime \prime}(1)(t-1)^{2}+\frac{1}{3 !} \psi^{\prime \prime \prime}(\xi)(\xi-1)^{3} \\
& =\frac{1}{2} \psi^{\prime \prime}(1)(t-1)^{2}+\frac{1}{3 !} \psi^{\prime \prime \prime}(\xi)(\xi-1)^{3}<\frac{p q+q+3}{2}(t-1)^{2}
\end{aligned}
$$

for some $\xi, 1 \leq \xi \leq t$. This completes the proof. 
LEMMA 3.3 ([5, Lemma 2.4]). If $\psi(t)$ satisfies Lemma 3.1(ii) and (iii), then $\psi(t)$ satisfies

$$
\psi^{\prime \prime}(t) \psi^{\prime}(\beta t)-\beta \psi^{\prime}(t) \psi^{\prime \prime}(\beta t)>0, \quad t>1, \beta>1 .
$$

REMARK 3.4. Let $\varphi(t)=\left(e^{p(g(t)-1)}-1\right) /(p q)$. Then $\psi(t)=\left(t^{2}-1\right) / 2+\varphi(t)$. Since $\varphi^{\prime}(t)=-e^{p(g(t)-1)} g(t) t^{-2}<0, \varphi(t)$ is monotonically decreasing with respect to $t>0$.

Let $\varrho:[0, \infty) \rightarrow[1, \infty)$ be the inverse function of $\psi(t)$ for $t \geq 1$ and $\rho:[0, \infty) \rightarrow(0,1]$ the inverse function of $-\psi^{\prime}(t) / 2$ for $t \in(0,1]$. Then we have the following lemma.

LEMMA 3.5. We have:

(i) $\sqrt{2 u+1} \leq \varrho(u) \leq 1+\sqrt{2 u}, u \geq 0$;

(ii) $\rho(z) \geq 1 /\left(1+q^{-1} \log \left(1+p^{-1} \log (1+2 z)\right)\right), z \geq 0$.

Proof. For (i), let $u=\psi(t)$ for $t \geq 1$. Then $\varrho(u)=t, t \geq 1$. Using Lemma 3.2(i), we have $u=\psi(t) \geq(t-1)^{2} / 2$. Then

$$
t=\varrho(u) \leq 1+\sqrt{2 u} .
$$

By the definition of $\varphi(t)$, we have $u=\psi(t)=\left(t^{2}-1\right) / 2+\varphi(t)$. Using Remark 3.4 and $\varphi(1)=0$, we have $\varphi(t) \leq 0, t \geq 1$. Hence,

$$
t^{2} / 2=u+1 / 2-\varphi(t) \geq u+1 / 2
$$

Thus

$$
t=\varrho(u) \geq \sqrt{2 u+1} .
$$

For (ii), let $z=-\psi^{\prime}(t) / 2$ for $0<t \leq 1$. Due to the definition of $\rho, \rho(z)=t$ for $z \geq 0$. From (3.3), we have $-t+e^{p(g(t)-1)} g(t) t^{-2}=2 z$ and

$$
e^{p(g(t)-1)} g(t) t^{-2}=t+2 z \leq 1+2 z, \quad 0<t \leq 1 .
$$

By taking the logarithm of both sides of (3.8), we obtain

$$
p(g(t)-1)+q\left(t^{-1}-1\right)-2 \log t \leq \log (1+2 z) .
$$

Since $0<t \leq 1$,

$$
g(t) \leq 1+p^{-1} \log (1+2 z) .
$$

Taking the logarithm of both sides of (3.9), we obtain

$$
q\left(t^{-1}-1\right) \leq \log \left(1+p^{-1} \log (1+2 z)\right)
$$

and hence

$$
t^{-1} \leq 1+q^{-1} \log \left(1+p^{-1} \log (1+2 z)\right) .
$$

That is,

$$
\rho(z)=t \geq \frac{1}{1+q^{-1} \log \left(1+p^{-1} \log (1+2 z)\right)} .
$$

This completes the proof. 
LEMmA 3.6. Let $\beta \geq 1$. Then $\psi(\beta t) \leq \psi(t)+t^{2}\left(\beta^{2}-1\right) / 2$.

PROOF. Using Remark 3.4, we have $\varphi(\beta t)-\varphi(t) \leq 0$ for $\beta \geq 1$. Hence

$$
\begin{aligned}
\psi(\beta t) & =\left((\beta t)^{2}-1\right) / 2+\varphi(\beta t) \\
& =\left(t^{2}-1\right) / 2+\varphi(t)+\left(\beta^{2} t^{2}-t^{2}\right) / 2+\varphi(\beta t)-\varphi(t) \\
& =\psi(t)+\left(t^{2} \beta^{2}-t^{2}\right) / 2+\varphi(\beta t)-\varphi(t) \\
& \leq \psi(t)+t^{2}\left(\beta^{2}-1\right) / 2 .
\end{aligned}
$$

This completes the proof.

Using Lemma 3.3, we have the following. The reader can refer to [5, Theorem 3.2] for the proof.

LEMMA 3.7. Let $\varrho:[0, \infty) \rightarrow[1, \infty)$ be the inverse function of $\psi(t), t \geq 1$. Then

$$
\Psi(\beta v) \leq n \psi(\beta \varrho(\Psi(v) / n)), \quad v \in \mathbf{R}_{++}^{\mathbf{n}}, \beta \geq 1 .
$$

In the following theorem we compute two upper bounds for the effect of a $\mu$-update on the value of $\Psi(v)$.

THEOREM 3.8. Let $0 \leq \theta<1$ and $v_{+}=v / \sqrt{1-\theta}$. If $\Psi(v) \leq \tau$, then:

(i) $\Psi\left(v_{+}\right) \leq(2 \tau+\theta \sqrt{8 n \tau}+\theta n) /(2(1-\theta))$;

(ii) $\Psi\left(v_{+}\right) \leq(\sqrt{n} \theta+\sqrt{2 \tau})^{2}(p q+q+3) /(2(1-\theta))$.

PROOF. For (i), using Lemma 3.6 with $\beta=1 / \sqrt{1-\theta}$, we obtain

$$
\Psi\left(v_{+}\right)=\Psi(\beta v) \leq \sum_{i=1}^{n}\left(\psi\left(v_{i}\right)+\frac{1}{2}\left(\beta^{2}-1\right) v_{i}^{2}\right)=\Psi(v)+\frac{\theta\|v\|^{2}}{2(1-\theta)} .
$$

By the first inequality in Lemma 3.2(i) and the Cauchy-Schwarz inequality,

$$
2 \Psi(v)=2 \sum_{i=1}^{n} \psi\left(v_{i}\right) \geq \sum_{i=1}^{n}\left(v_{i}-1\right)^{2}=\|v\|^{2}-2 e^{T} v+n \geq(\|v\|-\|e\|)^{2} .
$$

Hence,

$$
\|v\| \leq\|e\|+\sqrt{2 \Psi(v)}=\sqrt{n}+\sqrt{2 \Psi(v)} .
$$

From (3.10), (3.11), and $\Psi(v) \leq \tau$ we have

$$
\begin{aligned}
\Psi\left(v_{+}\right) & \leq \Psi(v)+\frac{\theta}{2(1-\theta)}(2 \Psi(v)+\sqrt{8 n \Psi(v)}+n) \\
& \leq \frac{1}{2(1-\theta)}(2 \tau+\theta \sqrt{8 n \tau}+\theta n) .
\end{aligned}
$$

For (ii), since $1 / \sqrt{1-\theta} \geq 1$ and $\varrho(\Psi(v) / n) \geq 1$, we have $\varrho(\Psi(v) / n) / \sqrt{1-\theta} \geq 1$. Using Lemma 3.7 with $\beta=1 / \sqrt{1-\theta}$, Lemma 3.2(ii), the second inequality in 
Lemma 3.5(i), and $\Psi(v) \leq \tau$,

$$
\begin{aligned}
\Psi\left(v_{+}\right) & \leq n \psi\left(\frac{\varrho(\Psi(v) / n)}{\sqrt{1-\theta}}\right) \leq \frac{(p q+q+3) n}{2}\left(\frac{\varrho(\Psi(v) / n)}{\sqrt{1-\theta}}-1\right)^{2} \\
& =\frac{(p q+q+3) n}{2}\left(\frac{\varrho(\Psi(v) / n)-\sqrt{1-\theta}}{\sqrt{1-\theta}}\right)^{2} \\
& \leq \frac{(p q+q+3) n}{2}\left(\frac{1+\sqrt{2 \tau / n}-\sqrt{1-\theta}}{\sqrt{1-\theta}}\right)^{2} \\
& \leq \frac{(p q+q+3) n}{2}\left(\frac{\theta+\sqrt{2 \tau / n}}{\sqrt{1-\theta}}\right)^{2} \\
& =\frac{p q+q+3}{2(1-\theta)}(\sqrt{n} \theta+\sqrt{2 \tau})^{2}
\end{aligned}
$$

where the last inequality holds because

$$
1-\sqrt{1-\theta}=\theta /(1+\sqrt{1-\theta}) \leq \theta, \quad 0<\theta<1 .
$$

This completes the proof.

Define

$$
\bar{\Psi}_{0}:=\frac{2 \tau+\theta \sqrt{8 n \tau}+\theta n}{2(1-\theta)}, \quad \tilde{\Psi}_{0}:=\frac{p q+q+3}{2(1-\theta)}(\sqrt{n} \theta+\sqrt{2 \tau})^{2} .
$$

We will use $\bar{\Psi}_{0}$ and $\tilde{\Psi}_{0}$ for the upper bounds of $\Psi(v)$ for large-update and small-update methods, respectively.

REMARK 3.9. By Theorem 3.8 and the assumption that $\Psi(v) \leq \tau$ just before the update of $\mu, \Psi\left(v_{+}\right) \leq \min \left\{\bar{\Psi}_{0}, \tilde{\Psi}_{0}\right\}$. For large-update methods with $\tau=\mathcal{O}(n)$ and $\theta=\Theta(1)$, we have $\overline{\bar{\Psi}}_{0}=\mathcal{O}(n)$, and for small-update methods with $\tau=\mathcal{O}(1)$ and $\theta=\Theta(1 / \sqrt{n})$, we have $\tilde{\Psi}_{0}=\mathcal{O}(p q+q)$.

\section{Complexity analysis}

In this section we compute a proper step size and the decrease of the proximity function during an inner iteration and give complexity results for the algorithm. For fixed $\mu$, if we take a step size $\alpha$, then we have a new iterate $x_{+}=x+\alpha \Delta x$, $s_{+}=s+\alpha \Delta s$. Using (2.5),

$$
x_{+}=x\left(e+\alpha \frac{\Delta x}{x}\right)=x\left(e+\alpha \frac{d_{x}}{v}\right)=\frac{x}{v}\left(v+\alpha d_{x}\right)
$$


and

$$
s_{+}=s\left(e+\alpha \frac{\Delta s}{s}\right)=s\left(e+\alpha \frac{d_{s}}{v}\right)=\frac{s}{v}\left(v+\alpha d_{s}\right) .
$$

Thus we have

$$
v_{+}:=\sqrt{\frac{x_{+} s_{+}}{\mu}}=\sqrt{\left(v+\alpha d_{x}\right)\left(v+\alpha d_{s}\right)} .
$$

Define, for $\alpha>0$,

$$
f(\alpha)=\Psi\left(v_{+}\right)-\Psi(v) .
$$

Then $f(\alpha)$ is the difference of proximities between a new iterate and a current iterate for fixed $\mu$. By Lemma 3.1(i),

$$
\Psi\left(v_{+}\right)=\Psi\left(\sqrt{\left(v+\alpha d_{x}\right)\left(v+\alpha d_{s}\right)}\right) \leq \frac{1}{2}\left(\Psi\left(v+\alpha d_{x}\right)+\Psi\left(v+\alpha d_{s}\right)\right) .
$$

Hence we have $f(\alpha) \leq f_{1}(\alpha)$, where

$$
f_{1}(\alpha):=\frac{1}{2}\left(\Psi\left(v+\alpha d_{x}\right)+\Psi\left(v+\alpha d_{s}\right)\right)-\Psi(v) .
$$

We have

$$
f(0)=f_{1}(0)=0 .
$$

Taking the derivative of $f_{1}(\alpha)$ with respect to $\alpha$, we obtain

$$
f_{1}^{\prime}(\alpha)=\frac{1}{2} \sum_{i=1}^{n}\left(\psi^{\prime}\left(v_{i}+\alpha\left[d_{x}\right]_{i}\right)\left[d_{x}\right]_{i}+\psi^{\prime}\left(v_{i}+\alpha\left[d_{s}\right]_{i}\right)\left[d_{s}\right]_{i}\right),
$$

where $\left[d_{x}\right]_{i}$ and $\left[d_{s}\right]_{i}$ denote the $i$ th components of the vectors $d_{x}$ and $d_{s}$, respectively. Using (3.5) and (3.7),

$$
f_{1}^{\prime}(0)=\frac{1}{2} \nabla \Psi(v)^{T}\left(d_{x}+d_{s}\right)=-\frac{1}{2} \nabla \Psi(v)^{T} \nabla \Psi(v)=-2 \delta^{2}(v) .
$$

Differentiating $f_{1}^{\prime}(\alpha)$ with respect to $\alpha$, we obtain

$$
f_{1}^{\prime \prime}(\alpha)=\frac{1}{2} \sum_{i=1}^{n}\left(\psi^{\prime \prime}\left(v_{i}+\alpha\left[d_{x}\right]_{i}\right)\left[d_{x}\right]_{i}^{2}+\psi^{\prime \prime}\left(v_{i}+\alpha\left[d_{s}\right]_{i}\right)\left[d_{s}\right]_{i}^{2}\right) .
$$

Since $f_{1}^{\prime \prime}(\alpha)>0, f_{1}(\alpha)$ is strictly convex in $\alpha$ unless $d_{x}=d_{s}=0$.

LEMMA 4.1. Let $\delta(v)$ be defined as in (3.7). Then

$$
\delta(v) \geq \sqrt{\Psi(v) / 2} .
$$

PROOF. Using Lemma 3.2(i),

$$
\delta(v)^{2}=\frac{1}{4}\|\nabla \Psi(v)\|^{2}=\frac{1}{4} \sum_{i=1}^{n}\left(\psi^{\prime}\left(v_{i}\right)\right)^{2} \geq \frac{\Psi(v)}{2} .
$$

Hence $\delta(v) \geq \sqrt{\Psi(v) / 2}$.

For notational convenience we denote $\delta:=\delta(v)$ and $\Psi:=\Psi(v)$. 
LEMMA 4.2 (Modification of [5, Lemma 4.1]). Let $f_{1}(\alpha)$ be defined as in (4.1) and $\delta$ as in (3.7). Then

$$
f_{1}^{\prime \prime}(\alpha) \leq 2 \delta^{2} \psi^{\prime \prime}\left(v_{\min }-2 \alpha \delta\right) .
$$

LEMMA 4.3 (Modification of [5, Lemma 4.2]). If the step size $\alpha$ satisfies the inequality

$$
-\psi^{\prime}\left(v_{\min }-2 \alpha \delta\right)+\psi^{\prime}\left(v_{\min }\right) \leq 2 \delta
$$

then

$$
f_{1}^{\prime}(\alpha) \leq 0
$$

Lemma 4.4 (Modification of [5, Lemma 4.3]). Let $\rho:[0, \infty) \rightarrow(0,1]$ denote the inverse function of $-\psi^{\prime}(t) / 2$ for all $t \in(0,1]$. Then, in the worst case, the largest step size $\bar{\alpha}$ satisfying (4.5) is given by

$$
\bar{\alpha}:=\frac{1}{2 \delta}(\rho(\delta)-\rho(2 \delta)) .
$$

Lemma 4.5 (Modification of [5, Lemma 4.4]). Let $\rho$ and $\bar{\alpha}$ be defined as in Lemma 4.4. Then

$$
\bar{\alpha} \geq \frac{1}{\psi^{\prime \prime}(\rho(2 \delta))} .
$$

For later reference, we define

$$
\tilde{\alpha}:=\frac{1}{\psi^{\prime \prime}(\rho(2 \delta))} .
$$

Then $\tilde{\alpha} \leq \bar{\alpha}$.

LEMMA 4.6 ([16, Lemma 1.3.3]). Suppose that $h(t)$ is a twice-differentiable convex function with

$$
h(0)=0, \quad h^{\prime}(0)<0,
$$

and such that $h(t)$ attains its (global) minimum at $t^{*}>0$, and $h^{\prime \prime}(t)$ is increasing with respect to $t$. Then for any $t \in\left[0, t^{*}\right]$,

$$
h(t) \leq \frac{t h^{\prime}(0)}{2}
$$

LEMMA 4.7 (Modification of [5, Lemma 4.5]). If the step size $\alpha$ is such that $\alpha \leq \tilde{\alpha}$, then

$$
f(\alpha) \leq-\alpha \delta^{2}
$$

PROOF. Let the univariate function $h$ be such that

$$
h(0)=f_{1}(0)=0, \quad h^{\prime}(0)=f_{1}^{\prime}(0)=-2 \delta^{2}, \quad h^{\prime \prime}(\alpha)=2 \delta^{2} \psi^{\prime \prime}\left(v_{\min }-2 \alpha \delta\right) .
$$

Then $h(t)$ is twice differentiable, $h(0)=0$, and $h^{\prime}(0)<0$. Since $h^{\prime \prime}(\alpha)>0, h(t)$ is strictly convex and hence has a global minimum at some $\alpha^{*}>0$. From (4.4), we have $f_{1}^{\prime \prime}(\alpha) \leq h^{\prime \prime}(\alpha)$. As a result, we have $f_{1}^{\prime}(\alpha) \leq h^{\prime}(\alpha)$ and $f_{1}(\alpha) \leq h(\alpha)$. Taking $\alpha \leq \tilde{\alpha}$ 
and using (4.5), we obtain

$$
\begin{aligned}
h^{\prime}(\alpha) & =h^{\prime}(0)+\int_{0}^{\alpha} h^{\prime \prime}(\zeta) d \zeta \\
& =-2 \delta^{2}+2 \delta^{2} \int_{0}^{\alpha} \psi^{\prime \prime}\left(v_{\min }-2 \xi \delta\right) d \xi \\
& =-2 \delta^{2}-\frac{2 \delta^{2}}{2 \delta} \int_{0}^{\alpha} \psi^{\prime \prime}\left(v_{\min }-2 \xi \delta\right) d\left(v_{\min }-2 \xi \delta\right) \\
& =-2 \delta^{2}-\delta\left(\psi^{\prime}\left(v_{\min }-2 \alpha \delta\right)-\psi^{\prime}\left(v_{\min }\right)\right) \\
& \leq-2 \delta^{2}+2 \delta^{2}=0 .
\end{aligned}
$$

Hence we have $\tilde{\alpha} \leq \alpha^{*}$. Since

$$
h^{\prime \prime \prime}(\alpha)=-4 \delta^{3} \psi^{\prime \prime \prime}\left(v_{\min }-2 \alpha \delta\right)>0,
$$

$h^{\prime \prime}(\alpha)$ is monotonically increasing in $\alpha$. Thus, using Lemma 4.6,

$$
f_{1}(\alpha) \leq h(\alpha) \leq \frac{\alpha h^{\prime}(0)}{2}=-\alpha \delta^{2} .
$$

Since $f(\alpha) \leq f_{1}(\alpha)$, the lemma is proved.

Using Lemma 4.7 and (4.6), we have the following lemma.

Lemma 4.8 (Modification of [5, Lemma 4.6]). Let $\tilde{\alpha}$ be defined as in (4.6). Then

$$
f(\tilde{\alpha}) \leq-\frac{\delta^{2}}{\psi^{\prime \prime}(\rho(2 \delta))} .
$$

Using Lemma 3.1(iv), we obtain the following.

LEMMA 4.9 (Modification of [5, Lemma 4.10]). The right-hand side in (4.7) is monotonically decreasing with respect to $\delta$.

Denote the value of $\Psi(v)$ after the $\mu$-update as $\Psi_{0}$. Then

$$
\Psi_{0} \leq \bar{\Psi}_{0} \quad \text { and } \quad \Psi_{0} \leq \tilde{\Psi}_{0},
$$

where $\bar{\Psi}_{0}$ and $\tilde{\Psi}_{0}$ are defined in (3.13). Define

$$
L(\Psi, p):=1+p^{-1} \log (1+2 \sqrt{2 \Psi}) .
$$

Then $L(\Psi, p)$ is monotonically increasing with respect to $\Psi$ and $L(\Psi, p) \geq 1$.

THEOREM 4.10. Let $\tilde{\alpha}$ be defined as in (4.6), $\mathfrak{X}:=1+q^{-1} \log (L(\Psi, p))$, and $\mathfrak{X}_{0}:=1+q^{-1} \log \left(L\left(\Psi_{0}, p\right)\right)$. Assume that $\Psi>\tau \geq 1$. Then for $p \geq 1$ and $q \geq 1$,

$$
f(\tilde{\alpha}) \leq-\frac{\sqrt{\Psi}}{10 L\left(\Psi_{0}, p\right) \mathfrak{X}_{0}^{4}\left(p q L\left(\Psi_{0}, p\right)+q+2\right)},
$$

where $L\left(\Psi_{0}, p\right):=1+p^{-1} \log \left(1+2 \sqrt{2 \Psi_{0}}\right)$. 
PROOF. Using Lemma 3.5(ii),

$$
\rho\left(2 \sqrt{\frac{\Psi}{2}}\right) \geq \frac{1}{1+q^{-1} \log \left(1+p^{-1} \log (1+2 \sqrt{2 \Psi})\right)} .
$$

Using (4.10), $\psi^{\prime \prime \prime}(t)<0$, for all $t>0,(3.3)$, and the assumption,

$$
\begin{aligned}
\psi^{\prime \prime}\left(\rho\left(2 \sqrt{\frac{\Psi}{2}}\right)\right) & \leq \psi^{\prime \prime}\left(\frac{1}{\mathfrak{X}}\right) \\
& =1+\left(p q L(\Psi, p)+q+\frac{2}{\mathfrak{X}}\right)(1+2 \sqrt{2 \Psi}) L(\Psi, p) \mathfrak{X}^{4} \\
& \leq 2(1+\sqrt{2 \Psi}) L(\Psi, p) \mathfrak{X}^{4}(p q L(\Psi, p)+q+2) \\
& \leq 5 \sqrt{\Psi} L(\Psi, p) \mathfrak{X}^{4}(p q L(\Psi, p)+q+2)
\end{aligned}
$$

where the last inequality follows from the assumption $\Psi \geq 1$ and a simple calculation. Using Lemmas 4.8, 4.9, 4.1, and (4.11),

$$
\begin{aligned}
f(\tilde{\alpha}) & \leq-\frac{\delta^{2}}{\psi^{\prime \prime}(\rho(2 \delta))} \\
& \leq-\frac{\Psi / 2}{\psi^{\prime \prime}(\rho(2 \sqrt{\Psi / 2}))} \\
& \leq-\frac{\Psi / 2}{5 \sqrt{\Psi} L(\Psi, p) \mathfrak{X}^{4}(p q L(\Psi, p)+q+2)} \\
& =-\frac{\sqrt{\Psi}}{10 L(\Psi, p) \mathfrak{X}^{4}(p q L(\Psi, p)+q+2)} \\
& \leq-\frac{\sqrt{\Psi}}{10 L\left(\Psi_{0}, p\right) \mathfrak{X}_{0}^{4}\left(p q L\left(\Psi_{0}, p\right)+q+2\right)},
\end{aligned}
$$

where the last inequality follows from the assumption $\Psi_{0} \geq \Psi$. This completes the proof.

LEMMA 4.11 ([15, Lemma 14]). Let $t_{0}, t_{1}, \ldots, t_{K}$ be a sequence of finitely many positive numbers such that

$$
t_{k+1} \leq t_{k}-\gamma t_{k}^{1-\tilde{\beta}}, \quad k=0,1, \ldots, K-1,
$$

where $\gamma>0$ and $0<\tilde{\beta} \leq 1$. Then $K \leq\left\lfloor t_{0}^{\tilde{\beta}} /(\gamma \tilde{\beta})\right\rfloor$.

After the $\mu$-update we denote the subsequent values in the same outer iteration as $\Psi_{k}, k=1,2, \ldots, K$. Then

$$
\Psi_{K-1}>\tau, \quad 0 \leq \Psi_{K} \leq \tau
$$


Denote

$$
N(\Psi, p, q):=L(\Psi, p)\left(1+q^{-1} \log (L(\Psi, p))\right)^{4}(p q L(\Psi, p)+q+2),
$$

where $L(\Psi, p)$ is defined in (4.9). Then $N(\Psi, p, q)$ is monotonically increasing with respect to $\Psi$.

LEMMA 4.12. Let $\bar{\Psi}_{0}$ and $\tilde{\Psi}_{0}$ be defined as in (3.13) and $K_{1}$ and $K_{2}$ be the total number of inner iterations in the outer iteration for large-update and small-update methods, respectively. Then for $p \geq 1$ and $q \geq 1$,

$$
K_{1} \leq 20 N\left(\bar{\Psi}_{0}, p, q\right) \bar{\Psi}_{0}^{1 / 2} \quad \text { and } \quad K_{2} \leq 20 N\left(\tilde{\Psi}_{0}, p, q\right) \tilde{\Psi}_{0}^{1 / 2} .
$$

Proof. By Theorem 4.10, Lemma 4.11 with $\tilde{\beta}:=1 / 2$ and $\gamma:=1 /\left(10 N\left(\Psi_{0}, p, q\right)\right)$, and (4.8),

$$
K_{1} \leq 20 N\left(\Psi_{0}, p, q\right) \Psi_{0}^{1 / 2} \leq 20 N\left(\bar{\Psi}_{0}, p, q\right) \bar{\Psi}_{0}^{1 / 2}
$$

and

$$
K_{2} \leq 20 N\left(\Psi_{0}, p, q\right) \Psi_{0}^{1 / 2} \leq 20 N\left(\tilde{\Psi}_{0}, p, q\right) \tilde{\Psi}_{0}^{1 / 2} .
$$

This completes the proof.

THEOREM 4.13. Let an LO be given and $\bar{\Psi}_{0}, \tilde{\Psi}_{0}$ be defined as in (3.13). Then for $p \geq 1, q \geq 1,0<\theta<1$, and $\tau \geq 1$, the total number of iterations required to obtain an approximate solution with $n \mu<\epsilon$ is bounded by:

(i) $\left\lceil(20 / \theta) N\left(\bar{\Psi}_{0}, p, q\right) \bar{\Psi}_{0}^{1 / 2} \log (n / \epsilon)\right\rceil$, for large-update methods;

(ii) $\left\lceil(20 / \theta) N\left(\tilde{\Psi}_{0}, p, q\right) \tilde{\Psi}_{0}^{1 / 2} \log (n / \epsilon)\right\rceil$, for small-update methods.

PROOF. If the parameter $\mu$ has the initial value $\mu^{0}=1$ and is updated by multiplying by $1-\theta$ with $0<\theta<1$, then after at most

$$
\lceil(1 / \theta) \log (n / \epsilon)\rceil
$$

iterations we have $n \mu<\epsilon$ [17]. For the total number of iterations, we multiply the number of inner iterations by the number of outer iterations. Hence the total numbers of iterations for large-update and small-update methods are bounded by

$$
\left\lceil(20 / \theta) N\left(\bar{\Psi}_{0}, p, q\right) \bar{\Psi}_{0}^{1 / 2} \log (n / \epsilon)\right\rceil \text { and }\left\lceil(20 / \theta) N\left(\tilde{\Psi}_{0}, p, q\right) \tilde{\Psi}_{0}^{1 / 2} \log (n / \epsilon)\right\rceil,
$$

respectively. This completes the proof.

REMARK 4.14. By Remark 3.9 and Theorem 4.13, for large-update methods, taking $\tau=\mathcal{O}(n)$ and $\theta=\Theta(1)$, the algorithm has

$$
\mathcal{O}\left(\mathfrak{Y}\left(1+q^{-1} \log (\mathfrak{Y})\right)^{4}(p q \mathfrak{Y}+q+2) \sqrt{n} \log (n / \epsilon)\right)
$$


iteration complexity where $\mathfrak{Y}:=1+p^{-1} \log (1+2 \sqrt{2 n})$. In particular, choosing $p=\mathcal{O}(\log (1+2 \sqrt{2 n}))$ and $q=1$, the algorithm has $\mathcal{O}(\sqrt{n} \log n \log (n / \epsilon))$ iteration complexity which is currently the best known result for large-update methods.

For small-update methods, taking $\tau=\mathcal{O}(1)$ and $\theta=\Theta(1 / \sqrt{n})$, the algorithm has

$$
\mathcal{O}\left(\mathfrak{Z}\left(1+q^{-1} \log (\mathfrak{Z})\right)^{4}(p q \mathfrak{Z}+q+2) \sqrt{n} \log (n / \epsilon)\right)
$$

iteration complexity where $\mathfrak{Z}:=1+p^{-1} \log (1+2 \sqrt{2(p q+q)})$. Choosing $p=1$ and $q=1$, we have $\mathcal{O}(\sqrt{n} \log (n / \epsilon))$ iteration complexity which is the best known complexity result for such methods.

\section{Concluding remarks}

Motivated by recent work of Amini and Haseli [1], we propose a new primaldual interior-point algorithm for LO problems based on a new kernel function and analyse the iteration complexity of the algorithm. We obtain $\mathcal{O}(\sqrt{n} \log n \log (n / \epsilon))$ and $\mathcal{O}(\sqrt{n} \log (n / \epsilon))$ iteration bounds for large-update and small-update methods, respectively. These are the best known results for such methods.

Future research might focus on the extension to semidefinite optimization, secondorder cone optimization, and complementarity problems. Numerical tests will be another topic for future research.

\section{Acknowledgements}

This work was supported by National Research Foundation of Korea grant funded by the Korean government (2010-0016200) and by 2010 Dongseo Frontier Project of Dongseo University. The authors would like to thank anonymous referees for their useful and kind comments which led to substantial improvement in the presentation of the paper.

\section{References}

[1] K. Amini and A. Haseli, "A new proximity function generating the best known iteration bounds for both large-update and small-update interior point methods", ANZIAM J. 49 (2007) 259-270.

[2] K. Amini and M. R. Peyghami, "Exploring complexity of large update interior-point methods for $P_{*}(\kappa)$ linear complementarity problem based on kernel function", Appl. Math. Comput. 207 (2009) 501-513.

[3] E. D. Andersen, J. Gondzio, Cs. Meszaros and X. Xu, "Implementation of interior point methods for large scale linear programming", in: Interior point methods of mathematical programming, (ed. T. Terlaky), (Kluwer Academic Publisher, Dordrecht, 1996) 189-252.

[4] Y. Q. Bai, M. El Ghami and C. Roos, "A new efficient large-update primal-dual interior-point method based on a finite barrier", SIAM J. Optim. 13 (2003) 766-782.

[5] Y. Q. Bai, M. El Ghami and C. Roos, "A comparative study of kernel functions for primal-dual interior-point algorithms in linear optimization”, SIAM J. Optim. 15 (2004) 101-128.

[6] Y. Q. Bai, G. Lesaja, C. Roos, G. Q. Wang and M. El Ghami, "A class of large-update and smallupdate primal-dual interior-point algorithms for linear optimization", J. Optim. Theory and Appl. 138 (2008) 341-359. 
[7] Y. Q. Bai and C. Roos, "A primal-dual interior point method based on a new kernel function with linear growth rate", Proceedings of the 9th Australian Optimization Day, Perth, Australia, 2002.

[8] Y. Q. Bai and C. Roos, "A polynomial-time algorithm for linear optimization based on a new simple kernel function”, Optim. Methods Software 18 (2003) 631-646.

[9] D. den Hertog, Interior point approach to linear, quadratic and convex programming, Volume 277 of Mathematics and its Applications (Kluwer Academic Publishers, Dordrecht, 1994).

[10] M. El Ghami, I. Ivanov, J. B. M. Melissen, C. Roos and T. Steihaug, "A polynomial-time algorithm for linear optimization based on a new class of kernel functions", J. Comput. Appl. Math. 224 (2009) 500-513.

[11] M. El Ghami and C. Roos, "Generic primal-dual interior point methods based on a new kernel function", RAIRO Oper. Res. 42 (2008) 199-213.

[12] C. C. Gonzaga, "Path following methods for linear programming", SIAM Rev. 34 (1992) 167-227.

[13] N. K. Karmarkar, "A new polynomial-time algorithm for linear programming", Combinatorica 4 (1984) 373-395.

[14] M. Kojima, S. Mizuno and A. Yoshise, "A primal-dual interior-point algorithm for linear programming", in: Progress in mathematical programming: interior point and related methods, (ed. N. Megiddo), (Springer, New York, 1989) 29-47.

[15] J. Peng, C. Roos and T. Terlaky, "Self-regular functions and new search directions for linear and semidefinite optimization", Math. Program. 93 (2002) 129-171.

[16] J. Peng, C. Roos and T. Terlaky, Self-regularity: a new paradigm for primal-dual interior-point algorithms (Princeton University Press, Princeton, NJ, 2002).

[17] C. Roos, T. Terlaky and J. Ph. Vial, Theory and algorithms for linear optimization: an interior approach (Wiley, Chichester, UK, 1997).

[18] G. Sonnevend, "An analytic center for polyhedrons and new classes of global algorithms for linear (smooth, convex) programming", System modeling and optimization: proceeding of the 12th IFIPconference, Budapest, Hungary, September 1985, Volume 84 of Lecture Notes in Control and Information Sciences (eds A. Prekopa, J. Szleezsan and B. Strazicky), (Springer, Berlin, 1986) $866-876$.

[19] N. J. Todd, "Recent developments and new directions in linear programming", in: Mathematical programming: recent developments and applications, (eds M. Iri and K. Tanabe), (Kluwer Academic Publishers, Dordrecht, 1989) 109-157. 